\title{
Investigating the microRNA-mRNA regulatory network in acute myeloid leukemia
}

\author{
HAIGUO ZHANG ${ }^{1,2^{*}}, \mathrm{CHENGFANG} \mathrm{ZHANG}^{3^{*}}$, RUI FENG $^{1,4^{*}}$, HAIXIA ZHANG $^{5}$, MIN GAO $^{3}$ and LING YE $^{2}$ \\ ${ }^{1}$ Department of Hematology, Qilu Hospital, Shandong University, Jinan, Shandong 250012; \\ Departments of ${ }^{2}$ Hematology and ${ }^{3}$ Clinical Laboratory, Jining No. 1 People's Hospital, Jining, Shandong 272011; \\ Departments of ${ }^{4}$ Hematology and ${ }^{5}$ Pharmacy, Yantai Yuhuangding Hospital, Yantai, Shandong 264000, P.R. China
}

Received November 27, 2015; Accepted May 25, 2017

DOI: $10.3892 / \mathrm{ol} .2017 .6686$

\begin{abstract}
Acute myeloid leukemia (AML) is a common myelogenous malignancy in adults that is often characterized by disease relapse. The pathophysiological mechanism of AML has not yet been elucidated. The present study aimed to identify the crucial microRNAs (miRNAs/miRs) and target genes in $A M L$, and to uncover the potential oncogenic mechanism of AML. miRNA and mRNA expression-profiling microarray datasets were downloaded from the Gene Expression Omnibus database. Differential expression analysis was performed and a regulatory network between miRNAs and target genes was constructed. Gene Ontology and Kyoto Encyclopedia of Genes and Genomes pathway enrichment analyses were used to predict the biological functions of the differentially expressed genes. Reverse transcription-quantitative polymerase chain reaction analysis was employed to verify the expression levels of miRNAs and target genes in AML patient samples. A total of 86 differentially expressed miRNAs and 468 differentially expressed mRNAs between AML and healthy blood samples were identified. In total, 47 miRNAs and 401 mRNAs were found to be upregulated, and 39 miRNAs and 67 mRNAs were found to be downregulated in AML. A total of 223 miRNA-target genes pairs were subjected to the construction of a regulatory network. Differentially expressed target genes were significantly enriched in the Wnt signaling pathway (hsa04310), melanogenesis (hsa04916) and pathways in cancer (hsa05200). Significantly differentially expressed miRNAs and genes, including hsa-miR-155, hsa-miR-192, annexin A2 (ANXA2), frizzled class receptor 3 (FZD3), and pleomorphic
\end{abstract}

Correspondence to: Dr Chengfang Zhang, Department of Clinical Laboratory, Jining No. 1 People's Hospital, 6 Jiankang Road, Jining, Shandong 272011, P.R. China

E-mail: chengfangzhang@126.com

*Contributed equally

Key words: acute myeloid leukemia, differential expression, microRNA, target genes, regulatory network, reverse transcription-quantitative polymerase chain reaction adenoma gene 1 (PLAG1), may serve essential roles in AML oncogenesis. Overall, hsa-miR-155, hsa-miR-192, ANXA2, FZD3 and PLAG1 may be associated with the development of AML via the involvement of the Wnt signaling pathway, melanogenesis and other cancer-associated signaling pathways.

\section{Introduction}

Leukemia is one of the 10 leading causes of cancer-associated mortality in China; in 2011 there were 27,907 mortalities in men and 19,708 mortalities in women from leukemia (1). The four types of Leukemia are acute lymphocytic leukemia, chronic lymphocytic leukemia, acute myeloid leukemia (AML) and chronic myeloid leukemia. AML accounts for $\sim 80 \%$ of cases of acute leukemia in adults (2).

AML is a highly heterogeneous leukemia associated with excessive progenitor cell proliferation and a differentiation block for cell-cycle arrest. AML is often caused by karyotypic abnormalities, including chromosomal translocations, deletions and inversions $(3,4)$. Etiological factors driving AML development remain unclear, but lifestyle and environmental exposures, including obesity and smoking, are reported to be associated with the disease (5).

The French-American-British (FAB) and World Health Organization (WHO) systems are the two main AML classification systems. The FAB system classifies AML into subtypes M0-M7 according to the cell type from which AML develops and the degree of maturation of the cells (6). According to the 2008 WHO Classification, AML are classified into six subgroups: AML with recurring genetic abnormalities, AML with myelodysplasia-related changes, therapy-related myeloid neoplasms, not otherwise specified AML, myeloid proliferations related to down syndrome and blastic plasmacytic dendritic cell neoplasms, with diagnosis performed according to morphology, cytochemistry, immunophenotype, genetics and clinical features (7).

Karyotypic abnormalities and genetic mutations are associated with AML progression and prognosis. Translocation of chromosomes 15 and 17 [t(15;17)], $\mathrm{t}(8 ; 21)$ or inversion of chromosome 16 is predictive of a relatively good prognosis (8), whereas deletion of chromosome 7 , deletion of $5 q$ or $>3$ chromosomal abnormalities is predictive of a poor prognosis in AML patients $(9,10)$. Fms-like tyrosine kinase 3-internal 
duplication (FLT3-ITD) and nucleophosmin (NPMI) are the two most commonly mutated genes in AML patients. Mutations to NPM1 occur in $50 \%$ of AML patients, whereas mutations to FLT3-ITD occur in $30 \%$. FLT3-ITD, KIT proto-oncogene receptor tyrosine kinase and brain and acute leukemia, cytoplasmic gene mutations have a negative impact on AML prognosis $(11,12)$, while NPMI and CCAAT/enhancer binding protein- $\alpha$ have a positive impact on prognosis (12-14).

At present, the pathogenic mechanism of AML is unclear. Acute promyelocytic leukemia (APL) is an M3 subtype of AML according to the FAB classification system. Overexpression of microRNA (miRNA/miR)-125a decreases APL NB4 cell proliferation, the inhibition of cell cycle progression and the promotion of cell apoptosis by targeting the ErbB pathway in APL (15). miR-150 expression induces the myeloid differentiation of human acute leukemia cells and normal hematopoietic progenitors. In AML patient samples and cell lines, miR-150 expression is low or absent, which contributes to the blocking of myeloid differentiation in acute leukemia cells (16).

The aim of the present study was to identify featured target genes of significantly differentially expressed miRNAs in AML by comparing AML samples with healthy ones, and analyzing the correlation of miRNA-target genes. Candidate target genes identified by these approaches may provide the groundwork for the elucidation of the mechanism of AML. However, further investigation of the potential function of these genes in the treatment of AML is required.

\section{Materials and methods}

Transcriptomics datasets. In the Gene Expression Omnibus (GEO; http://ncbi.nlm.nih.gov/geo/) (17), only the studies comparing AML and healthy blood were assessed. A total of 6 studies were assessed in which the global profile of gene expression was measured in AML patients' blood samples, with accession numbers GSE48558, GSE35008, GSE35010, GSE24395, GSE17054 and GSE51908. The details of studies, including the platform, number of cases, controls, year and author, were extracted and assessed.

Data processing and identification of differentially expressed miRNAs and mRNAs. Raw expression datasets were downloaded from the GEO and the raw datasets were preprocessed by $\log _{2}$ transformation and Z-score normalization. Limma, which is a linear model for microarray data analysis, was utilized to analyze the differentially expressed miRNAs and mRNAs between the AML and healthy control samples (18). A false discovery rate (FDR) of $<0.05$ was set as the threshold of differentially expressed miRNAs and mRNAs.

miRNA target gene prediction. Targets genes for differentially expressed miRNAs were predicted via miRTarBase (http://mirtarbase.mbc.nctu.edu.tw/). Over 50,000 miRNA-target interactions in the miRTarBase database have been validated by experiments such as reporter assays, western blotting or microarray experiments with overexpression or knockdown of miRNAs $(19,20)$.

Construction of regulatory miRNA-mRNA networks. The miRNA-mRNA interaction network of differentially expressed miRNA and mRNA was visualized using Cytoscape (http://cytoscape.org) (21). This software presents the regulation between miRNA and mRNA as two-dimensional network with nodes and edges, which represent miRNA-target gene associations.

Functional enrichment analysis of the differentially expressed target genes. To obtain the functions of differentially expressed targeted genes, Gene Ontology (GO) terms (22) and Kyoto Encyclopedia of Genes and Genomes (KEGG) (23) pathways were enriched using GOEAST (http://omicslab.genetics. ac.cn/GOEAST) (24) and GeneCodis (http://genecodis.cnb .csic.es/analysis), respectively (25). $\mathrm{P}<0.01$ and FDR $<0.05$ were set as the thresholds of significance for GO terms and KEGG pathway analysis.

Reverse transcription-quantitative polymerase chain reaction (RT-qPCR). The blood samples were collected from 3 males with AML treated in Qilu Hospital of Shandong University (Shandong, China) in 2015, with a mean age of 45.6 years. In addition, 3 normal blood samples were also included with corresponding gender and age. Total RNA of fresh blood samples were extracted by TRIzol reagent (Invitrogen; Thermo Fisher Scientific, Inc., Waltham, MA, USA) according to the manufacturer's instructions. Use of these samples was approved by the Ethics Committee of Qilu Hospital of Shandong University (Jinan, China). The SuperScript III Reverse Transcription kit (Invitrogen; Thermo Fisher Scientific, Inc.) was used to synthesize the cDNA according to the manufacturer's instructions. RT-qPCR was performed using Power SYBR Green PCR Master mix (Applied Biosystems; Thermo Fisher Scientific, Inc.) on the Applied Biosystems 7500 (Applied Biosystems; Thermo Fisher Scientific, Inc.). The RT-qPCR cycling conditions were 1 cycle of $95^{\circ} \mathrm{C}$ for $10 \mathrm{~min}$, followed by 45 cycles of $95^{\circ} \mathrm{C}$ for $15 \mathrm{sec}$ and $60^{\circ} \mathrm{C}$ for $60 \mathrm{sec}$. The miRcute miRNA First-Strand cDNA kit (Tiangen Biotech Co., Ltd., Bejing, China) and the miRcute miRNA qPCR Detection kit (Tiangen Biotech Co., Ltd.) were used for miRNA expression level detection. The RT-qPCR cycling conditions for miRNA were 1 cycle of $94^{\circ} \mathrm{C}$ for $2 \mathrm{~min}$, followed by 45 cycles of $94^{\circ} \mathrm{C}$ for $20 \mathrm{sec}$ and $60^{\circ} \mathrm{C}$ for $34 \mathrm{sec}$. U6 small nuclear RNA and $\beta$-actin was used as internal controls for miRNA and mRNA detection, respectively. The relative expression of target genes was calculated using the $2^{-\Delta \Delta \mathrm{Cq}}$ method (26). At least three independent experiments were performed. The PCR primers used were as follows: hsa-miR-155 forward, 5'-TAATGCTAATCG TGATAGGGGT-3' and reverse, GTGCAGGGTCCGAGGT; hsa-miR-192 forward, 5'-TGACCTATGAATTGACAGCC-3' and reverse, GTGCAGGGTCCGAGGT; frizzled class receptor 3 (FZD3) forward, 5'-TCTCCTCTTAGCTGGCAT TATATCC-3' and reverse, 5'-GCAGCGTTCTTGTATCCA CGTT-3'; and Annexin A2 (ANXA2) forward, 5'-AGAATC ATGGTCTCCCGCAGTG-3' and reverse, 5'-TCCACCACA CAGGTACAGCAGC-3'.

Statistical analysis. RT-qPCR experimental data was expressed as the mean \pm standard deviation. Statistical significance was evaluated using an unpaired Student's t-test. $\mathrm{P}<0.05$ was considered to indicate a statistically significant difference. 
Table I. Characteristics of mRNA and miRNA expression profiling of the acute myeloid leukemia.

A, mRNA expression profiling

\begin{tabular}{|c|c|c|c|c|}
\hline Author, year & $\begin{array}{l}\text { Gene expression } \\
\text { omnibus ID }\end{array}$ & Platform & $\begin{array}{l}\text { Samples, } \\
\text { H:P }\end{array}$ & (Refs.) \\
\hline Civin et al, 2013 & GSE48558 & $\begin{array}{l}\text { GPL6244 [HuGene-1_0-st] Affymetrix } \\
\text { Human Gene 1.0 ST Array [transcript } \\
\text { (gene) version] }\end{array}$ & $49: 18$ & $(27)$ \\
\hline Barreyro et al, 2012 & GSE35008 & $\begin{array}{l}\text { GPL6244 [HuGene-1_0-st] Affymetrix } \\
\text { Human Gene 1.0 ST Array }\end{array}$ & $16: 12$ & $(28)$ \\
\hline Barreyro et al, 2012 & GSE35010 & $\begin{array}{l}\text { GPL6244 [HuGene-1_0-st] Affymetrix } \\
\text { Human Gene 1.0 ST Array }\end{array}$ & $16: 15$ & $(28)$ \\
\hline Kikushige et al, 2010 & GSE24395 & $\begin{array}{l}\text { GPL6106 Sentrix Human-6 v2 Expression } \\
\text { BeadChip }\end{array}$ & $5: 12$ & (29) \\
\hline Majeti et al, 2009 & GSE17054 & $\begin{array}{l}\text { GPL570 [HG-U133_Plus_2] Affymetrix } \\
\text { Human Genome U133 Plus 2.0 Array }\end{array}$ & $4: 9$ & (30) \\
\hline
\end{tabular}

B, miRNA expression profiling

\begin{tabular}{lccc}
\hline Author, year & $\begin{array}{c}\text { Gene expression } \\
\text { omnibus ID }\end{array}$ & Platform & $\begin{array}{c}\text { Samples, } \\
\text { H:P } \\
\text { (Refs.) }\end{array}$ \\
\hline Tan et al, 2013 & GSE51908 & $\begin{array}{l}\text { GPL8786 [miRNA-1_0] Affymetrix } \\
\text { miRNA Array }\end{array}$ & (31)
\end{tabular}

H, healthy subject; P, AML patient; miRNA, microRNA.

\section{Results}

Differentially expressed miRNAs and mRNAs in AML. A total of 5 mRNA and 1 miRNA expression profiles datasets, including 137 AML and 84 healthy samples were downloaded from the GEO, normalized and processed (Table I) (27-31). Differentially expressed genes between AML and normal samples, including 86 miRNAs and 468 mRNAs, were screened with a threshold of FDR $<0.05$. Of the 86 miRNAs, 47 were upregulated and 39 were downregulated in AML samples compared with the normal samples; of the 468 mRNAs, 401 were upregulated and genes 67 were downregulated. The top 10 upregulated and downregulated miRNAs are shown in Table II (the full list of differentially expressed miRNAs and mRNAs is not shown).

Construction of miRNA-mRNA regulatory networks. The miRTarBase database was used to predict the target genes of the 47 upregulated and 39 downregulated miRNAs in AML; 223 miRNA-target gene pairs, including 31 differentially expressed miRNAs and 153 target genes, were visualized using Cytoscape software (Fig. 1). A total of 55 differentially expressed miRNAs, including hsa-miR-29b-1* and hsa-miR-194, were not displayed in the network, as the 55 differentially expressed miRNAs were not available in miRTarBase database (data not shown). hsa-miR-26b, hsa-miR-192, hsa-miR-21, hsa-miR-181a and hsa-miR-155 regulated 43 , 25, 26, 15 and 11 targets, respectively, and displayed the highest connectivity. Pleomorphic adenoma gene 1 (PLAGl), high-mobility group AT-hook 2, RUN-domain-containing 3B, transmembrane protein $2, \mathrm{TNF}-\alpha$ induced protein 3 and family with sequence similarity 3 member $C$, which were regulated by $7,5,4,4,4$ and 4 miRNAs, respectively, were the mRNAs with the highest connectivity (Fig. 1).

Functional analysis of miRNA target genes. GO classification and KEGG pathway analyses were used to obtain the biological functions of miRNA target genes, including biological process, cellular component, molecular function and signaling pathway. The threshold of GO classification was set as $\mathrm{P}<0.01$. Negative regulation of blood coagulation (GO:0030195, $\mathrm{P}=1.83 \times 10^{-24}$ ), negative regulation of hemostasis (GO:1900047, $\mathrm{P}=1.83 \times 10^{-24}$ ) and negative regulation of coagulation (GO:0050819, $\mathrm{P}=2.65 \times 10^{-23}$ ) were the most significantly enriched target genes of biological processes; sarcolemma (GO:0042383, $\mathrm{P}=1.85 \times 10^{-29}$ ), Schmidt-Lanterman incisure (GO:0043220, $\mathrm{P}=1.80 \times 10^{-25}$ ) and myelin sheath adaxonal region (GO:0035749, $\mathrm{P}=5.91 \times 10^{-25}$ ) were the most significantly enriched target genes of the cellular component; and phospholipase inhibitor activity (GO:0004859, $\left.\mathrm{P}=1.14 \times 10^{-44}\right)$, lipase inhibitor activity (GO:0055102, $\left.\mathrm{P}=3.76 \times 10^{-43}\right)$ and calcium-dependent phospholipid binding (GO:0005544, $\mathrm{P}=5.77 \times 10^{-41}$ ) were the most significantly enriched target genes of the molecular function (Table III).

In total, 148 of the 153 differentially expressed miRNA target genes were enriched in the KEGG database. The Wnt signaling pathway $\left(\mathrm{FDR}=8.70 \times 10^{-4}\right)$, melanogenesis $\left(\mathrm{FDR}=8.70 \times 10^{-4}\right)$ and pathways in cancer $\left(\mathrm{FDR}=1.60 \times 10^{-3}\right)$ 


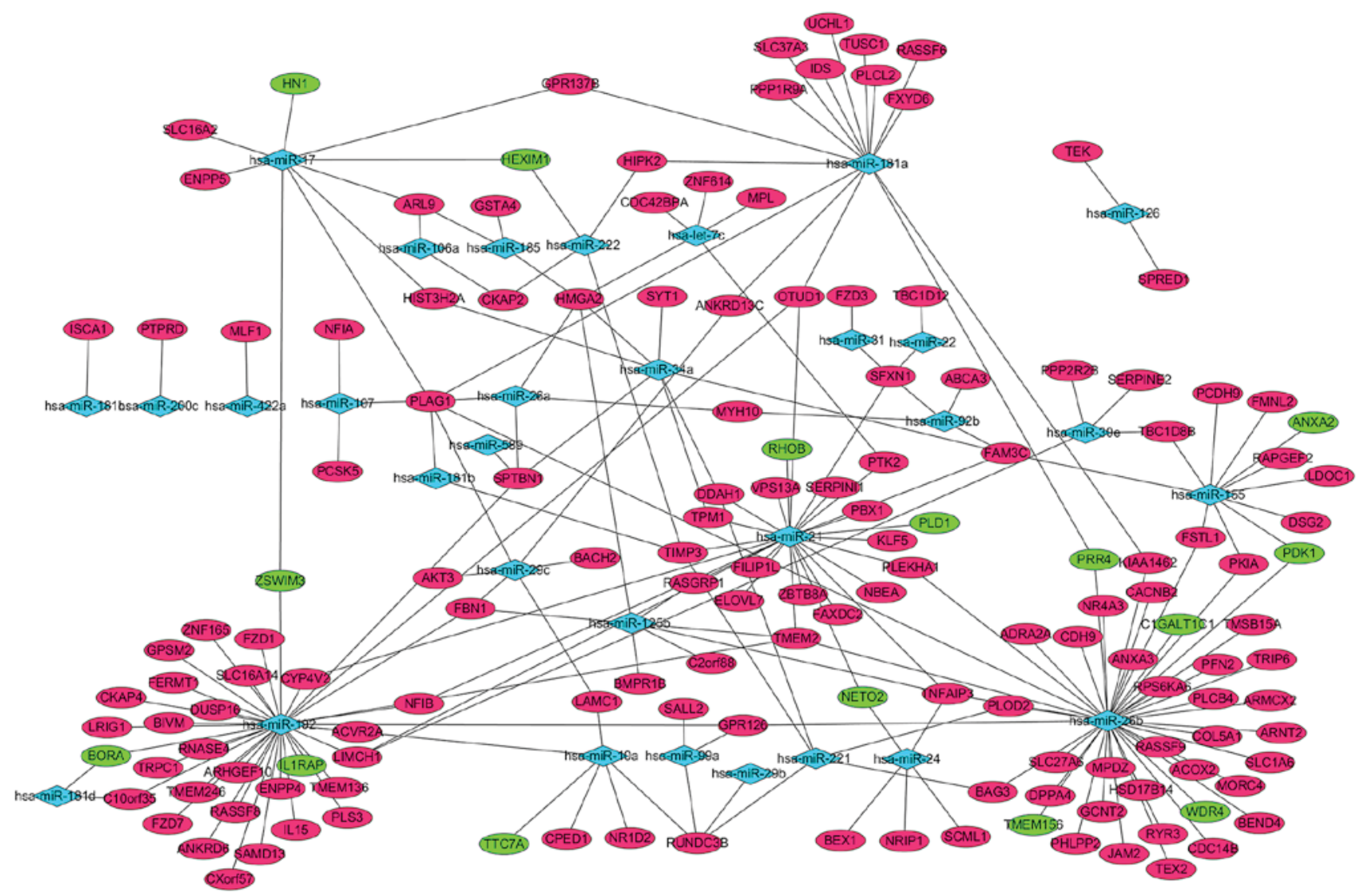

Figure 1. miRNA-target gene regulatory network of acute myeloid leukemia. Circular nodes represent target genes and diamond nodes represent miRNAs. Green nodes represent downregulation, red nodes represent upregulation. Solid lines indicate regulatory associations between the miRNAs and target genes. miRNA/miR, microRNA.

were the most significantly enriched pathways in KEGG analysis, with the criteria of FDR $<0.05$ (Table IV).

$R T$-qPCR validation of differentially expressed miRNAs and target genes. To validate the microarray analysis data, the levels of significant differentially expressed miRNA and target genes were quantified by RT-qPCR in three AML blood samples and three normal blood samples. hsa-miR-155 was significantly $(\mathrm{P}<0.05)$ upregulated in AML compared with that in the normal samples, and the target gene $A N X A 2$ was significantly downregulated in AML (Fig. 2A). FZD3 was significantly upregulated in the three AML samples compared with the normal samples $(\mathrm{P}<0.01$; Fig. $2 \mathrm{~B})$. The present study identified hsa-miR-192 as a downregulated miRNA in AML, although the expression level was not found to be significantly different in AML by RT-qPCR validation (Fig. 2C).

\section{Discussion}

In the present study, hsa-miR-155 was one of the five miRNAs with the highest connectivity with target genes, targeting 11 differentially expressed mRNAs (Fig. 1), and was significantly upregulated in AML. In the present study, ANXA2 was predicted as a putative target gene of hsa-miR-155. RT-qPCR validated that hsa-miR-155 was significantly upregulated and ANXA2 was significantly downregulated in AML (Fig. 2A), which is in accordance with the bioinformatics analysis.
The fact that hsa-miR-155 was upregulated in AML was consistent with the results of a previous study (32). Mounting evidence identifies hsa-miR-155 as having an oncogenic role, generating AML; overexpression of hsa-miR-155 causes myeloproliferation with cell cell-cycle arrest $(33,34)$. High expression of hsa-miR-155 is associated with a poor outcome in AML patients, which has been observed in numerous AML patients via sequencing studies and miRNA expression analyses (35-37). Additionally, hsa-miR-155 is reported to contribute to the metastasis of various solid tumors, including colorectal carcinoma (38), oral squamous cell carcinoma (39) and renal cell carcinoma (40). ANXA2 is a target gene of hsa-miR-155 and its downregulation is associated with a poor AML patient prognosis, based on gene expression profile analysis (41). hsa-miR-155 upregulation and ANXA2 downregulation may be potential biomarkers for the clinical evaluation of AML prognosis.

Through KEGG analysis, FZD3 was found to be enriched in four signaling pathways, including the Wnt signaling pathway, melanogenesis, pathways in cancer and basal cell carcinoma. The Wnt signaling pathway was the most significantly enriched pathway in AML (Table IV). Higher expression of FZD3 was detected in three AML patients compared with that in the normal control, as determined by RT-qPCR (Fig. 2B), which was consistent with the bioinformatics analysis. FZD3 is a member of the frizzled gene family, which also includes $F Z D 1$ and $F Z D 7$, and functions as 
Table II. Significantly differentially expressed miRNAs (top 10).

\begin{tabular}{|c|c|c|}
\hline miRNA & P-value & $\begin{array}{c}\text { Log } \\
\text { (fold-change) }\end{array}$ \\
\hline \multicolumn{3}{|c|}{ Upregulated miRNAs } \\
\hline hsa-miR-432 & $9.93 \times 10^{-12}$ & 1.66 \\
\hline hsa-miR-126 & $7.44 \times 10^{-10}$ & 1.57 \\
\hline hsa-miR-10a & $4.35 \times 10^{-8}$ & 1.55 \\
\hline hsa-miR-130a & $3.39 \times 10^{-11}$ & 1.54 \\
\hline hsa-miR-34a & $2.05 \times 10^{-14}$ & 1.43 \\
\hline hsa-miR-181d & $2.32 \times 10^{-13}$ & 1.3 \\
\hline hsa-miR-181a* & $6.65 \times 10^{-10}$ & 1.3 \\
\hline hsa-miR-551b* & $3.27 \times 10^{-8}$ & 1.17 \\
\hline hsa-miR-501-5p & $1.17 \times 10^{-8}$ & 1.08 \\
\hline hsa-miR-125b & $6.04 \times 10^{-5}$ & 1.06 \\
\hline \multicolumn{3}{|c|}{ Downregulated miRNAs } \\
\hline hsa-miR-192 & $6.74 \times 10^{-7}$ & -1.12 \\
\hline hsa-miR-29b-1* & $2.75 \times 10^{-8}$ & -1.1 \\
\hline hsa-miR-194 & $1.66 \times 10^{-5}$ & -1.1 \\
\hline hsa-miR-31 & $2.98 \times 10^{-3}$ & -1.05 \\
\hline hsa-miR-26b & $6.59 \times 10^{-8}$ & -0.971 \\
\hline hsa-miR-628-3p & $6.31 \times 10^{-4}$ & -0.755 \\
\hline hsa-miR-30e & $2.84 \times 10^{-4}$ & -0.715 \\
\hline hsa-miR-29b & $1.53 \times 10^{-4}$ & -0.664 \\
\hline hsa-miR-200c & $3.06 \times 10^{-5}$ & -0.635 \\
\hline hsa-miR-21 & $3.96 \times 10^{-3}$ & -0.605 \\
\hline
\end{tabular}

miRNA/miR, microRNA.

a receptor for the canonical Wnt/ $/$-catenin signaling pathway. Overactivation of the Wnt signaling pathway contributes to tumorigenesis $(42,43)$. According to the present study, the Wnt signaling pathway was essential for AML progression and oncogenicity. CXXC finger protein 5, which is frequently deleted in AML, inhibits the Wnt pathway and leukemic cell proliferation (44). Activation of the $\mathrm{Wnt} / \beta$-catenin pathway mediates transformation of AML progenitor cells and results in impaired myelomonocytic differentiation $(45,46)$. The FZD3/Wnt signaling pathway may therefore be important in AML pathogenesis.

In the present study, hsa-miR-192 was the most significantly downregulated miRNA and regulated 25 target genes in AML (Fig. 1). miR-192 downregulation is associated with cell cycle progression, cell growth, apoptosis and proliferation of solid tumors $(47,48)$. Overexpression of miR-192 induces apoptotic death in bladder cancer cells, increases the proportion of cells in the G0/G1 phase and decreases the proportion of cells in the $\mathrm{S}$ phase compared with a control (47). Curcumin is a traditional Chinese medicine extracted from turmeric that inhibits non-small cell lung cancer cell (NSCLC) cell proliferation and induces NSCLC cell apoptosis through the upregulation of miR-192-5p and the suppression of the phosphoinositide-3 kinase/protein kinase B signaling pathway $(47,48)$. In the present study, hsa-miR-192
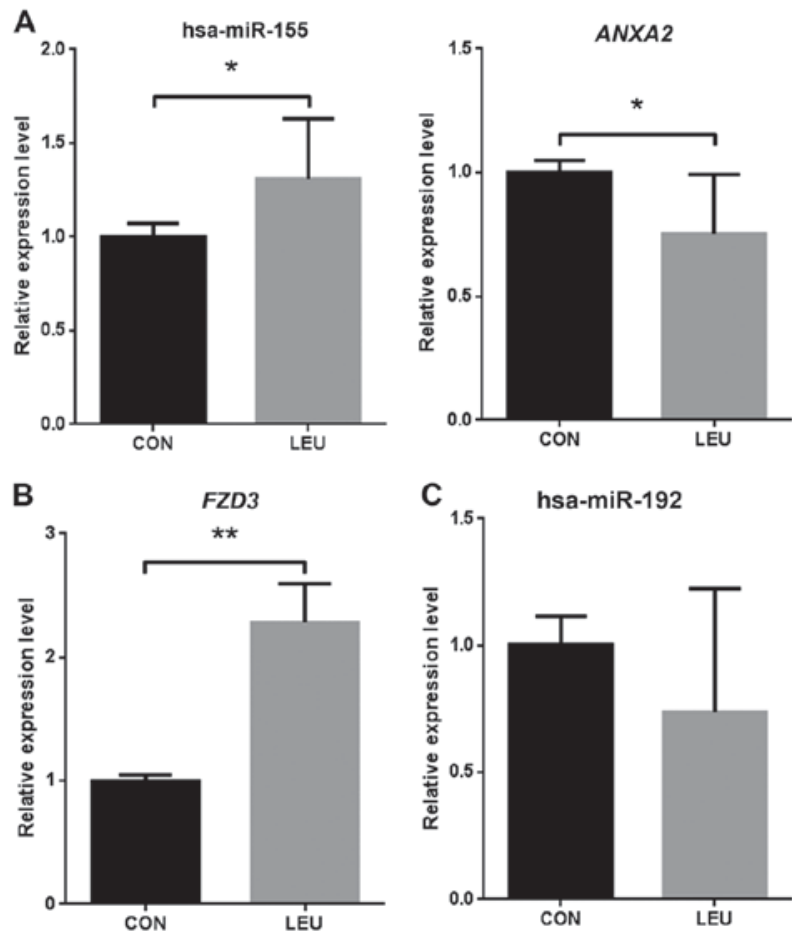

Figure 2. Verification of miRNA and target gene expression levels in AML and normal controls, as determined by reverse transcription-quantitative polymerase chain reaction. (A) hsa-miR-155 and $A N X A 2$ expression levels in AML patients and healthy controls. (B) FZD3 expression levels in AML patients and healthy controls. (C) hsa-miR-192 expression levels in AML patients and healthy controls. ${ }^{*} \mathrm{P}<0.05,{ }^{* *} \mathrm{P}<0.01$. miRNA/miR, microRNA; AML, acute myeloid leukemia; ANXA2, annexin A2; FZD3, frizzled class receptor 3; CON, healthy control patient blood samples; LEU, AML patient blood samples.

was downregulated in AML (Fig. 2C), suggesting that it may also serve a key role in AML cell apoptosis and proliferation.

PLAGl was targeted by 7 miRNAs, meaning it had the highest connectivity of the mRNAs in the miRNA-mRNA network (Fig. 1). The PLAG family consists of 3 members (PLAG1, PLAGL1 and PLAGL2), each with a highly conserved zinc finger structure that allows them to function as transcription factors to recognize DNA and/or RNA (49). PLAG1 serves an oncogenic role in AML, cooperating with CBF-SMMHC to induce AML tumorigenesis (50). The results of the present study revealed that PLAG1 was upregulated in AML.

In summary, a miRNA-mRNA regulatory network was constructed based on differentially expressed miRNAs and target genes in AML. In this network, a number of miRNAs and target genes that may play important roles in AML, such as hsa-miR-155, hsa-miR192, ANXA2, FZD3 and PLAG1, were identified. These results indicated that the Wnt signaling pathway, melanogenesis and pathways in cancer may be involved in the pathogenesis of AML. An miRNA-target gene regulatory network was constructed in AML using bioinformatic tools. A number of miRNAs and mRNAs that are potentially important for AML tumorigenesis were identified. However, the mechanism behind the associations between miRNA, mRNA and miRNA-mRNA involved in AML progression and development requires further investigation. 
Table III. GO annotation of differentially expressed microRNA target genes in acute myeloid leukemia samples (top 15).

\begin{tabular}{l} 
GO ID \\
\hline Biological process \\
GO:0030195 \\
GO:1900047 \\
GO:0050819 \\
GO:0042730 \\
GO:0040023 \\
GO:0051961 \\
GO:0051964 \\
GO:0030198 \\
GO:0043062 \\
GO:0051241 \\
GO:0001525 \\
GO:0060252 \\
GO:0030320 \\
GO:0030644 \\
GO:0055064
\end{tabular}

Cellular component

GO:0042383
GO:0043220
GO: 0035749
GO: 0043218
GO:0005925
GO:0005924
GO:0030055
GO:0070161
GO:0005912
GO:0043209
GO:0019897
GO:0019898
GO:0030054
GO:0014704
GO:0044291

Molecular function

GO:0004859

GO:0055102

GO:0005544

GO:0030234

GO:0004857

GO:0005509

GO:0005546

GO:0005543

GO:1901981

GO:0008289

GO:0043548

GO:0008092

GO:0017137

GO:0004713

GO:0035091

Count

P-value

Negative regulation of blood coagulation

Negative regulation of hemostasis

Negative regulation of coagulation

Fibrinolysis

Establishment of nucleus localization

Negative regulation of nervous system development

Negative regulation of synapse assembly

Extracellular matrix organization

Extracellular structure organization

Negative regulation of multicellular organismal process

Angiogenesis

Positive regulation of glial cell proliferation

Cellular monovalent inorganic anion homeostasis

Cellular chloride ion homeostasis

Chloride ion homeostasis

Sarcolemma

Schmidt-Lanterman incisure

Myelin sheath adaxonal region

Compact myelin

Focal adhesion

Cell-substrate adherens junction

Cell-substrate junction

Anchoring junction

Adherens junction

Myelin sheath

Extrinsic to plasma membrane

Extrinsic to membrane

Cell junction

Intercalated disc

Cell-cell contact zone

21

21

21

17

16

14

14

35

35
35

35

40

35

15

14

14

14

33

18

17

18

30

30

30

32

31

18

18

18
18

18
40

14

14

Phospholipase inhibitor activity

Lipase inhibitor activity

Calcium-dependent phospholipid binding

Enzyme regulator activity

Enzyme inhibitor activity

Calcium ion binding

Phosphatidylinositol-4,5-bisphosphate binding

Phospholipid binding

Phosphatidylinositol phosphate binding

Lipid binding

Phosphatidylinositol 3-kinase binding

Cytoskeletal protein binding

Rab GTPase binding

Protein tyrosine kinase activity

Phosphatidylinositol binding
29

29

35

79

43

65

18

53

19

56

14

51

17

25

22

$1.83 \times 10^{-24}$

$1.83 \times 10^{-24}$

$2.65 \times 10^{-23}$

$1.90 \times 10^{-22}$

$2.55 \times 10^{-22}$

$2.70 \times 10^{-22}$

$2.70 \times 10^{-22}$

$6.31 \times 10^{-21}$

$6.58 \times 10^{-21}$

$2.08 \times 10^{-20}$

$2.86 \times 10^{-20}$

$3.64 \times 10^{-20}$

$3.68 \times 10^{-20}$

$3.68 \times 10^{-20}$

$3.68 \times 10^{-20}$

$1.85 \times 10^{-29}$

$1.80 \times 10^{-25}$

$5.91 \times 10^{-25}$

$2.95 \times 10^{-23}$

$1.69 \times 10^{-21}$

$3.04 \times 10^{-21}$

$1.30 \times 10^{-20}$

$1.09 \times 10^{-17}$

$1.73 \times 10^{-17}$

$2.16 \times 10^{-15}$

$2.01 \times 10^{-13}$

$4.02 \times 10^{-10}$

$1.61 \times 10^{-09}$

$2.10 \times 10^{-09}$

$3.13 \times 10^{-09}$

$1.14 \times 10^{-44}$

$3.76 \times 10^{-43}$

$5.77 \times 10^{-41}$

$1.58 \times 10^{-23}$

$1.15 \times 10^{-22}$

$2.23 \times 10^{-20}$

$2.28 \times 10^{-20}$

$7.37 \times 10^{-19}$

$2.81 \times 10^{-17}$

$1.54 \times 10^{-15}$

$6.81 \times 10^{-14}$

$1.35 \times 10^{-13}$

$1.55 \times 10^{-13}$

$1.43 \times 10^{-12}$

$2.75 \times 10^{-11}$

GO, Gene Ontology. 
Table IV. KEGG pathway enrichment analysis of differentially expressed microRNA target genes in acute myeloid leukemia (top 15).

\begin{tabular}{|c|c|c|c|c|}
\hline KEGG ID & KEGG term & Count & FDR & Genes \\
\hline hsa04310 & Wnt signaling pathway & 4 & $8.70 \times 10^{-4}$ & FZD7, PLCB4, FZD1, FZD3 \\
\hline hsa04916 & Melanogenesis & 4 & $8.70 \times 10^{-4}$ & FZD7, PLCB4, FZD1, FZD3 \\
\hline hsa05200 & Pathways in cancer & 8 & $1.60 \times 10^{-3}$ & $\begin{array}{l}\text { FZD7, AKT3, FZD1, LAMC1, } \\
\text { FZD3, PTK2, ARNT2, PLD1 }\end{array}$ \\
\hline hsa05146 & Amoebiasis & 4 & $2.65 \times 10^{-3}$ & PLCB4, LAMC1, PTK2, COL5A1 \\
\hline hsa05222 & Small cell lung cancer & 3 & $2.90 \times 10^{-3}$ & AKT3, LAMC1, PTK2 \\
\hline hsa04010 & MAPK signaling pathway & 6 & $3.04 \times 10^{-3}$ & $\begin{array}{l}\text { DUSP16, RASGRP1, RPS6KA6, } \\
\text { RAPGEF } 2, A K T 3, C A C N B 2\end{array}$ \\
\hline hsa05217 & Basal cell carcinoma & 3 & $3.59 \times 10^{-3}$ & FZD7, FZD1, FZD3 \\
\hline hsa04724 & Glutamatergic synapse & 4 & $4.75 \times 10^{-3}$ & SLC1A6, PLCB4, TRPC1, PLD1 \\
\hline hsa04530 & Tight junction & 4 & $5.01 \times 10^{-3}$ & JAM2, MYH10, AKT3, MPDZ \\
\hline hsa04630 & Jak-STAT signaling pathway & 4 & $8.08 \times 10^{-3}$ & IL15, AKT3, MPL, SPRED1 \\
\hline hsa04060 & Cytokine-cytokine receptor interaction & 5 & $9.00 \times 10^{-3}$ & $\begin{array}{l}\text { IL15, BMPRIB, MPL, ILIRAP, } \\
\text { ACVR } 2 A\end{array}$ \\
\hline hsa04660 & T-cell receptor signaling pathway & 3 & $1.57 \times 10^{-2}$ & RASGRP1, AKT3, PDK1 \\
\hline hsa04510 & Focal adhesion & 4 & $1.61 \times 10^{-2}$ & AKT3, LAMC1, PTK2, COL5A1 \\
\hline hsa04722 & Neurotrophin signaling pathway & 3 & $2.02 \times 10^{-2}$ & RPS6KA6, AKT3, PDK1 \\
\hline hsa05145 & Toxoplasmosis & 3 & $2.12 \times 10^{-2}$ & $A K T 3, L A M C 1, P D K 1$ \\
\hline
\end{tabular}

KEGG, Kyoto Encyclopedia of Genes and Genomes; FDR, false discovery rate.

\section{Acknowledgements}

The present study was supported by a grant from the Program of Jining Science and Technology Development Plan (grant no, 2015-57-102).

\section{References}

1. Chen W, Zheng R, Zeng $\mathrm{H}$ and Zhang S: The updated incidences and mortalities of major cancers in China, 2011. Chin J Cancer 34: 507-507, 2015.

2. Cripe LD: Adult acute leukemia. Curr Probl Cancer 21: 1-64, 1997.

3. Marcucci G, Haferlach T and Döhner H: Molecular genetics of adult acute myeloid leukemia: Prognostic and therapeutic implications. J Clin Oncol 29: 475-486, 2011.

4. Deguchi K and Gilliland DG: Cooperativity between mutations in tyrosine kinases and in hematopoietic transcription factors in AML. Leukemia 16: 740-744, 2002.

5. Finn L, Sproat L, Heckman MG, Jiang L, Diehl NN, Ketterling R, Tibes R, Valdez R and Foran J: Epidemiology of adult acute myeloid leukemia: Impact of exposures on clinical phenotypes and outcomes after therapy. Cancer Epidemiol 39: 1084-1092, 2015.

6. Bennett JM, Catovsky D, Daniel MT, Flandrin G, Galton DA, Gralnick HR and Sultan C: Proposals for the classification of the acute leukaemias. French-American-British (FAB) co-operative group. Br J Haematol 33: 451-458, 1976.

7. Vardiman JW, Thiele J, Arber DA, Brunning RD, Borowitz MJ, Porwit A, Harris NL, Le Beau MM, Hellström-Lindberg E, Tefferi A and Bloomfield CD: The 2008 revision of the World Health Organization (WHO) classification of myeloid neoplasms and acute leukemia: Rationale and important changes. Blood 114: 937-951, 2009.

8. Marcucci G, Mrózek K, Ruppert AS, Maharry K, Kolitz JE, Moore JO, Mayer RJ, Pettenati MJ, Powell BL, Edwards CG, et al: Prognostic factors and outcome of core binding factor acute myeloid leukemia patients with $\mathrm{t}(8 ; 21)$ differ from those of patients with inv(16): A Cancer and Leukemia Group B study. J Clin Oncol 23: 5705-5717, 2005.
9. Stone RM: Prognostic factors in AML in relation to (ab)normal karyotype. Best Pract Res Clin Haematol 22: 523-528, 2009.

10. Schanz J and Haase D: Cytogenetic features in myelodysplastic syndromes. 2014.

11. Care RS, Valk PJM, Goodeve AC, Abu-Duhier FM, Geertsma-Kleinekoort WM, Wilson GA, Gari MA, Peake IR, Löwenberg B and Reilly JT: Incidence and prognosis of c-KIT and FLT3 mutations in core binding factor (CBF) acute myeloid leukaemias. Br J Haematol 121: 775-777, 2003.

12. Langer C, Radmacher MD, Ruppert AS, Whitman SP, Paschka P, Mrózek K, Baldus CD, Vukosavljevic T, Liu CG, Ross ME, et al: High BAALC expression associates with other molecular prognostic markers, poor outcome, and a distinct gene-expression signature in cytogenetically normal patients younger than 60 years with acute myeloid leukemia: A Cancer and Leukemia Group B (CALGB) study. Blood 111: 5371-5379, 2008.

13. Preudhomme C, Sagot C, Boissel N, Cayuela JM, Tigaud I, de Botton S, Thomas X, Raffoux E, Lamandin C, Castaigne S, et al: Favorable prognostic significance of CEBPA mutations in patients with de novo acute myeloid leukemia: A study from the acute leukemia French association (ALFA). Blood 100: 2717-2723, 2002.

14. Döhner K, Tobis K, Ulrich R, Fröhling S, Benner A, Schlenk RF and Döhner H: Prognostic significance of partial tandem duplications of the MLL gene in adult patients 16 to 60 years old with acute myeloid leukemia and normal cytogenetics: A study of the acute myeloid leukemia study group Ulm. J Clin Oncol 20: 3254-3261, 2002.

15. Ufkin ML, Peterson S, Yang X, Driscoll H, Duarte C and Sathyanarayana P: miR-125a regulates cell cycle, proliferation, and apoptosis by targeting the ErbB pathway in acute myeloid leukemia. Leuk Res 38: 402-410, 2014.

16. Morris VA, Zhang A, Yang T, Stirewalt DL, Ramamurthy R, Meshinchi S and Oehler VG: MicroRNA-150 expression induces myeloid differentiation of human acute leukemia cells and normal hematopoietic progenitors. PLoS One 8: e75815, 2013.

17. Edgar R, Domrachev M and Lash AE: Gene expression omnibus: NCBI gene expression and hybridization array data repository. Nucleic Acids Res 30: 207-210, 2002.

18. Diboun I, Wernisch L, Orengo CA and Koltzenburg M: Microarray analysis after RNA amplification can detect pronounced differences in gene expression using limma. BMC Genomics 7: 252, 2006. 
19. Hsu SD, Lin FM, Wu WY, Liang C, Huang WC, Chan WL, Tsai WT, Chen GZ, Lee CJ, Chiu CM, et al: miRTarBase: A database curates experimentally validated microRNA-target interactions. Nucleic Acids Res 39 (Database Issue): D163-D169, 2011.

20. Hsu SD, Tseng YT, Shrestha S, Lin YL, Khaleel A, Chou CH Chu CF, Huang HY, Lin CM, Ho SY, et al: miRTarBase update 2014: An information resource for experimentally validated miRNA-target interactions. Nucleic Acids Res 42 (Database Issue): D78-D85, 2014.

21. Shannon P, Markiel A, Ozier O, Baliga NS, Wang JT, Ramage D, Amin N, Schwikowski B and Ideker T: Cytoscape: A software environment for integrated models of biomolecular interaction networks. Genome Res 13: 2498-2504, 2003.

22. Cheng $\mathrm{L}$, Lin $\mathrm{H}, \mathrm{Hu} \mathrm{Y}$, Wang $\mathrm{J}$ and Yang Z: Gene function prediction based on the Gene Ontology hierarchical structure. PLoS One 9: e107187, 2014.

23. Kanehisa M, Sato Y, Kawashima M, Furumichi M and Tanabe M: KEGG as a reference resource for gene and protein annotation. Nucleic Acids Res 44: D457-D462, 2016.

24. Zheng Q and Wang XJ: GOEAST: A web-based software toolkit for Gene Ontology enrichment analysis. Nucleic Acids Res 36: W358-W363, 2008.

25. Carmona-Saez $\mathrm{P}$, Chagoyen M, Tirado F, Carazo JM and Pascual-Montano A: GENECODIS: A web-based tool for finding significant concurrent annotations in gene lists. Genome Biol 8 . R3, 2007.

26. Livak KJ and Schmittgen TD: Analysis of relative gene expression data using real-time quantitative PCR and the 2(-Delta Delta C(T)) method. Methods 25: 402-408, 2001

27. Cramer-Morales K, Nieborowska-Skorska M, Scheibner K, Padget M, Irvine DA, Sliwinski T, Haas K, Lee J, Geng H, Roy D, et al: Personalized synthetic lethality induced by targeting RAD52 in leukemias identified by gene mutation and expression profile. Blood 122: 1293-1304, 2013.

28. Barreyro L, Will B, Bartholdy B, Zhou L, Todorova TI,Stanley RF, Ben-Neriah S, Montagna C, Parekh S, Pellagatti A, et al: Overexpression of IL-1 receptor accessory protein in stem and progenitor cells and outcome correlation in AML and MDS Blood 120: 1290-1298, 2012.

29. Kikushige Y, Shima T, Takayanagi S, Urata S, Miyamoto T, Iwasaki $\mathrm{H}$, Takenaka $\mathrm{K}$, Teshima $\mathrm{T}$, Tanaka $\mathrm{T}$, Inagaki $\mathrm{Y}$ and Akashi K: TIM-3 is a promising target to selectively kill acute myeloid leukemia stem cells. Cell Stem Cell 7: 708-717, 2010.

30. Majeti R, Becker MW, Tian Q, Lee TL, Yan X, Liu R, Chiang JH, Hood L, Clarke MF and Weissman IL: Dysregulated gene expression networks in human acute myelogenous leukemia stem cells. Proc Natl Acad Sci USA 106: 3396-3401, 2009.

31. Tan YS, Kim M, Kingsbury TJ, Civin CI and Cheng WC: Regulation of RAB5C is important for the growth inhibitory effects of MiR-509 in human precursor-B acute lymphoblastic leukemia. PLoS One 9: e111777, 2014.

32. Havelange V, Stauffer N, Heaphy CC, Volinia S, Andreeff M, Marcucci G, Croce CM and Garzon R: Functional implications of microRNAs in acute myeloid leukemia by integrating microRNA and messenger RNA expression profiling. Cancer 117: 4696-4706, 2011.

33. Forrest AR, Kanamori-Katayama M, Tomaru Y, Lassmann T, Ninomiya N, Takahashi $\mathrm{Y}$, de Hoon MJ, Kubosaki A, Kaiho A, Suzuki M, et al: Induction of microRNAs, mir-155, mir-222, mir-424 and mir-503, promotes monocytic differentiation through combinatorial regulation. Leukemia 24: 460-466, 2010.

34. O'Connell RM, Rao DS, Chaudhuri AA, Boldin MP, Taganov KD, Nicoll J, Paquette RL and Baltimore D: Sustained expression of microRNA-155 in hematopoietic stem cells causes a myeloproliferative disorder. J Exp Med 205: 585-594, 2008
35. Marcucci G, Maharry KS, Metzeler KH, Volinia S, Wu YZ, Mrózek K, Nicolet D, Kohlschmidt J, Whitman SP, Mendler JH, et al: Clinical role of microRNAs in cytogenetically normal acute myeloid leukemia: miR-155 upregulation independently identifies high-risk patients. J Clin Oncol 31: 2086-2093, 2013.

36. Chuang MK, Chiu YC, Chou WC, Hou HA, Chuang EY and Tien HF: A 3-microRNA scoring system for prognostication in de novo acute myeloid leukemia patients. Leukemia 29: $1051-1059,2015$

37. Zhi F, Cao X, Xie X, Wang B, Dong W, Gu W, Ling Y, Wang R, Yang Y and Liu Y: Identification of circulating microRNAs as potential biomarkers for detecting acute myeloid leukemia. PLoS One 8: e56718, 2013

38. Qu YL, Wang HF, Sun ZQ, Tang Y, Han XN, Yu XB and Liu K: Up-regulated miR-155-5p promotes cell proliferation, invasion and metastasis in colorectal carcinoma. Int J Clin Exp Pathol 8: 6988-6994, 2015.

39. Baba O, Hasegawa S, Nagai H, Uchida F, Yamatoji $M$, Kanno NI, Yamagata K, Sakai S, Yanagawa T and Bukawa H: MicroRNA-155-5p is associated with oral squamous cell carcinoma metastasis and poor prognosis. J Oral Pathol Med 45: 248-255, 2016

40. Merhautova J, Hezova R, Poprach A, Kovarikova A, Radova L, Svoboda M, Vyzula R, Demlova R and Slaby O: miR-155 and miR-484 are associated with time to progression in metastatic renal cell carcinoma treated with sunitinib. Biomed Res Int 2015: 941980, 2015

41. Park MH, Cho SA, Yoo KH, Yang MH, Ahn JY, Lee HS, Lee KE, Mun YC, Cho DH, Seong CM and Park JH: Gene expression profile related to prognosis of acute myeloid leukemia. Oncol Rep 18: 1395-1402, 2007.

42. Conacci-Sorrell M, Zhurinsky J and Ben-Ze'ev A: The cadherin-catenin adhesion system in signaling and cancer. J Clin Invest 109: 987-991, 2002

43. Giles RH, van Es JH and Clevers H: Caught up in a Wnt storm: Wnt signaling in cancer. Biochim Biophys Acta 1653: $1-24,2003$.

44. Kühnl A, Valk PJ, Sanders MA, Ivey A, Hills RK, Mills KI, Gale RE, KaiserMF, Dillon R, Joannides M, et al: Downregulation of the Wnt inhibitor CXXC5 predicts a better prognosis in acute myeloid leukemia. Blood 125: 2985-2994, 2015.

45. Wang Y, Krivtsov AV, Sinha AU, North TE, Goessling W, Feng Z, Zon LI and Armstrong SA: The Wnt/beta-catenin pathway is required for the development of leukemia stem cells in AML. Science 327: 1650-1653, 2010.

46. Simon M, Grandage VL, Linch DC and Khwaja A: Constitutive activation of the Wnt/beta-catenin signalling pathway in acute myeloid leukaemia. Oncogene 24: 2410-2420, 2005.

47. Ye M, Zhang J, Zhang J, Miao Q, Yao L and Zhang J: Curcumin promotes apoptosis by activating the p53-miR-192-5p/215-XIAP pathway in non-small cell lung cancer. Cancer Lett 357: 196-205, 2015.

48. Jin H, Qiao F, Wang Y, Xu Y and Shang Y: Curcumin inhibits cell proliferation and induces apoptosis of human non-small cell lung cancer cells through the upregulation of miR-192-5p and suppression of PI3K/Akt signaling pathway. Oncol Rep 34: 2782-2789, 2015.

49. Kas K, Voz ML, Hensen K, Meyen E and Van de Ven WJ: Transcriptional activation capacity of the novel PLAG family of zinc finger proteins. J Biol Chem 273: 23026-23032, 1998.

50. Landrette SF, Kuo YH, Hensen K, Barjesteh van Waalwijk van Doorn-Khosrovani S, Perrat PN, Van de Ven WJ, Delwel R and Castilla LH: Plag1 and Plagl2 are oncogenes that induce acute myeloid leukemia in cooperation with Cbfb-MYH11. Blood 105: 2900-2907, 2005. 